\title{
Measuring perceptions of students toward game and recreational activity using fuzzy conjoint analysis
}

\author{
Siti Siryani Sofian, Azmin Sham Rambely \\ Department of Mathematical Sciences, Faculty of Science \& Technology, Universiti Kebangsaan Malaysia, Malaysia
}

\begin{tabular}{l}
\hline \hline Article Info \\
\hline Article history: \\
Received Dec 19, 2019 \\
Revised Mar 11, 2020 \\
Accepted Apr 6, 2020 \\
\hline
\end{tabular}

\section{Keywords:}

Conjoint analysis

Fuzzy sets

Mathematics education

Multi-criteria problem

\begin{abstract}
The effectiveness of game and recreational activity (GaRA) can be determined from the application of fuzzy conjoint analysis (FCA). The analysis was used due to fuzziness in determining individual perceptions. This study involved a survey collected from 1494 students who participated in a Mathematics Discovery Camp organized by a UKM research group. The aim of this research is to determine the effectiveness of modules delivered to cultivate affection and confidence in Mathematics through different factors. There were 11 games conducted for the participants, and their perceptions based on the evaluation of six attributes were measured. A seven-point Likert scale method representing seven linguistic terms was used to collect students' preferences to indicate their perceptions of each GaRA module. Scores of perceptions were transformed into degrees of similarity. Results found that interest, effort and team work were the strongest values obtained from GaRA as participants strongly agreed that these attributes fulfilled their preferences in most module. The evaluation using fuzzy conjoint model implicated the successfulness of a fuzzy approach to evaluate fuzziness obtained in the Likert-scale, and has shown its ability in ranking the attributes from most preferred to least preferred.
\end{abstract}

Copyright $(0) 2020$ Institute of Advanced Engineering and Science. All rights reserved.

\section{Corresponding Author:}

Siti Siryani Sofian,

Center of Modelling \& Data Science,

Faculty of Science and Technology,

Universiti Kebangsaan Malaysia,

43600 Bangi, Selangor Malaysia.

Email: seeryani@yahoo.com

\section{INTRODUCTION}

Students' engagement and interest toward Mathematics subject is a situation to be concerned. This can be seen from the statement by Senior Fellow of Malaysia Science Academy, Datuk Dr. Abdul Aziz Sheikh Abdul Kadir, as he expected the increment of the number of Science, Technology, Engineering and Mathematics (STEM) students, but only $20 \%$ of Malaysian students followed STEM in school. The former Minister of Science, Technology and Innovation, Datuk Seri Madius Tangau, stated that the lack of support from teachers and students' perception of Mathematics as a boring subject are the reasons toward difficulty to achieve the increment of number of STEM students. The presence of students' anxiety in the learning process such as anxiety toward mathematics assessments, temporality, understanding problem, numbers and mathematical operations and anxiety toward Mathematics in real life situations are the factors that generate negative impacts toward students' learning [1]. The challenges in learning Mathematics are stronger, and therefore, it is necessary to solve the problem by finding the new method of teaching and learning process that stimulates students' interest. Mathematical anxiety of interest and negative feeling toward Mathematics are factors that make Mathematics difficult to learn. Besides that, lack of prior knowledge, motivation, and counseling create misunderstanding and problems to study Mathematics [2]. 
Students' performance in Mathematics depends on their perceptions. Strengths and weaknesses in Mathematics, learning material, interest in Mathematics, difficulties or challenges in doing Mathematics, self-confidence and beliefs about Mathematics are identified as the construction of perceptions that influence students' performance. The negative perception of Mathematics is the most crucial problem that lowers students' motivation. The main reasons for hating Mathematics are difficulty in understanding the subject, poor instruction, and demand more time to understand [3]. Mathematics is considered a difficult subject by most students due to the aversive teaching style, difficulty in following instruction, difficulty in understanding the subject, difficulty in remembering its equations, and ways to solve problems. There is a strong association between their belief regarding the difficulty of subjects and dislike toward Mathematics. Perception of Mathematics as a difficult subject is associated strongly to lower self-efficacy on the subject. Therefore, it is necessary to counter the problem immediately by encouraging students' involvement in the learning process, as their involvement in learning process is directly proportional to their interest [4]. The integration of fun learning process and infusion of confidence is the first step toward students' involvement in class.

Creating a culture of achievement in the classroom, developing interactive and relevant lessons and activities, and being encouraging and supportive to students are very important in order to foster students' engagement in the classroom. A study by [5] shows that students' engagement is directly proportional to their performance. There are three types of class engagements. Firstly, class engagement which refers to students' investment in their own learning to determine their needs and enjoyment of mental difficulties. Second, emotional engagement which involves students' responses to the learning system involving teachers, peers, and course contents. Lastly, behavioral engagement including students' participation in learning, their effort, and attendance. Learning through playing and activity is an effective learning method that can foster students' engagement [6]. Playing provides a valuable social context such that the interaction with more knowledgeable or experienced others can be promoted. The social context creates a situation where innovation, risk taking, and creative problem solving are involved. As learning through playing is believed to be an effective learning method that can create students' engagement, game and recreational activity (GaRA) is introduced to cultivate students' affection and confidence toward Mathematics [7, 8]. GaRA is a problem based learning approach that challenges students to learn through engagement in real-life problems. In GaRA, interactive and relevant lessons are presented to the students. It is a group activity and facilitated by adults who play an active role by assessing students's understanding, generating situations, asking questions, introducing elements of surprise, and working with students to consider the logical consequences of the position they adopt. Module content, facilitators, and group members play an important role as a situational interest which then produces a development of individual interest. It is very important to determine how effective GaRA is in developing students' engagement in the learning process, so that they can overcome their anxiety toward Mathematics. Feedbacks on and perceptions of modules in GaRA are important elements to identify its effectiveness, and the evaluation of students' perceptions is determined through fuzzy conjoint analysis.

The theory of conjoint measurement is a general theory of continuous quantity. The fundamental measurement was introduced at least in 1920s [9, 10]. However, conjoint measurement was discovered in 1964 by a mathematical psychologist, Luce, and a statistician, Tukey, written in a seminal paper [11]. Thereafter, various studies in theoretical measurement and choice-based conjoint were developed. Scales for dependent and independent variables exist under conjoint measurement condition. Prior to 1970, works by [11] on conjoint measurement might have been applied in marketing problems, consumer evaluations, preference analysis, and similarity judgments. Conjoint analysis is a statistical method to determine a value that an individual places on features of products or services. It is a multi-criteria evaluation to determine the preferences of individuals with respect to criteria considered. It is normally used in market researches, but is also available in any multi-criteria problems in the science field [12]. Studies in consumer judgment are very important, as feedbacks from consumers are significant to ensure the survival of an organization in terms of products and services. It is also important in product management and marketing analysis, where firms will be able to develop and price their products according to market preference [13]. This shows that conjoint analysis plays an important role, as it is an optimal market research for measuring a value that consumers place on features of a product or service.

However, due to its fuzziness in determining individual perceptions, fuzzy set is introduced to analyze and evaluate the fuzziness or vagueness. Fuzzy logic starts with a fuzzy set, a set without a crisp. It contains elements with only a partial degree of membership, for example, the shades of blue. Most of us agree that denim, ultramarine, and dark blue belong to blue type, but what about turquoise or teal? It feels like a part of blue, but somehow it seems like it should technically be excluded as it is also a part of shades of green. Individual perceptions and cultural background must be taken into account when defining shades of color. Fuzzy reasoning becomes valuable when looking at how people really perceive the concept of colors. 
Since perceptions are fuzzy matter, fuzzy reasoning has major advantage in determining individuals' preference [14-16] as it offers the ability to reply to a yes-no question to not-quite-yes-or-no answer. This ability allows us to evaluate human language and contributes to understand individual preference [17]. Many studies have proposed to integrate fuzzy theory and conjoint analysis to estimate the product or service preferences. This model is proposed by [18] to allow fuzzy theory in predicting consumers' choice by combining the vector conjoint model with fuzzy measurement in a new model that requires ordinal data. This model has extended its application in surveys developed in education [17], students' preference [7, 8], operational and management [19], and often on behalf of marketing [20]. The effectiveness of this model comes with an understanding on the relationship between customer value and optimization of services in order to give the best balance of quality, and to maximize values that the customers get.

In this study, we applied [18] fuzzy conjoint model (FCM) for the evaluation of students' perceptions of GaRA. Fuzzy set conjoint analysis is able to evaluate students' perceptions in the form of degree of membership, and could be used significantly as analysis of learning outcomes [21]. Students' perceptions are a collection of students' negative and positive feedbacks toward a program, and their perceptions are one of the basic factors that is significant in determining the effectiveness of GaRA, as we believed that GaRA approaches show better outcomes in student's confidence and performance in Mathematics. After completing each game, students were asked to respond to a survey containing six statements. Each statement represents an attribute. These attributes are considered as the factors that create affection and build confidence in Mathematics. The six statements which represent six attributes are shown in Table 1.

Table 1. The six statements in linguistic term and their membership function

\begin{tabular}{lccc}
\hline & Statements & Attributes & \\
\hline 1. & I like this activity & Interest & A1 \\
2. & This activity caused me to love Mathematics & Passion & A2 \\
3. & I can solve this activity & Solving skills & A3 \\
4. & I put my effort to solve this activity & Effort & A4 \\
5. & Team work encouraged me to solve this activity & Team work & A5 \\
6. & Reward encouraged me to solve this activity & Extrinsic motivation & A6 \\
\hline
\end{tabular}

This study measures students' affection and confidence toward Mathematics through the perceptions based on the evaluation of the six attributes. Affection is determined by students' interest and passion, while their level of confidence is measured from their problem solving skill, effort, team work and their responses on rewards.

\section{RESEARCH METHOD}

\subsection{Fuzzy theory}

Fuzzy set is a mathematical model of vague data. It is introduced by [22] who intends to deal with vague information. Such set is characterized by a membership function in an interval [0 1]. Zadeh defines fuzzy set by considering $X$ to be a space of points, with a generic element of $X$ denoted by $x$. Thus, $X=\{x\}$. He also defines that a fuzzy set $A$ in $X$ is characterized by a membership function $f_{A}(x)$ which associates each point in $X$ in an interval [0 1]. A value of $f_{A}(x)$ at $x$ represents the "grade of membership function" of $x$ in $A$. In a classical theory of set, the membership function can take on only two values which are 0 and 1 , with $f_{A}(x)=1$ as $x$ belongs to $A$, and 0 if $x$ does not belong to $A$. The application of fuzzy sets in decision making process is crucial in our efforts to evaluate multiple conflicting criteria in decision making. Decision making in a fuzzy environment is a decision process in which the goals or constraints are fuzzy in nature [23]. Therefore, fuzzy sets are important for handling various forms of uncertainty with a multi-criteria character of many optimization problems.

A study by [24] consideres Bellman-Zadeh approach to the analysis of a fuzzy set. The subsequent process of decision making is associated with the reduction of the problems to multi-criteria decision making using fuzzy preference relation. Fuzzy logic dealing with approximated reasoning compared to exact reasoning, and solves the problems of modeling preferences in terms of fuzzy binary relations [25]. This is a better technique compared to the probabilistic technique because the general idea of traditional approach is selecting the highest probability [26]. However, human preference is vague, and decisions based on Likert discretization are imperfect. The best way is to put a membership degree in the interval $0 \leq f_{A}(x) \leq 1$ to determine the level of confidence that $x$ satisfies the property $X$. Fuzzy set theory has the ability to evaluate human language which is impossible to do using classical set theory [27]. Human language in the form of words or sentences is used as a motivation to be less specific than numerical ones. For example, the term set of age might be read as,

\footnotetext{
Measuring perceptions of students toward game and recreational activity using fuzzy... (Siti Siryani Sofian)
} 
$T($ age $)=\{$ young, not young, very young, not very young, ..., middle-age, not middle-age,..., old, not old, ... $\}$.

By characterizing the set by words, it serves as an approximate characterization of a phenomenon which is too complex to be described in precise term. However, existing mathematical apparatus of system can be adapted to the manipulation of the linguistic variable such as fuzzy logic. In the case of a linguistic variable for age, the numerical base value is the numbers, for example, $0,1,2, \ldots, 100$. A fuzzy restriction on the value of the base variable is characterized by a compatibility function in the interval $[0,1]$. For example, the compatibilities of the numerical age 22,28 , and 35 with a young restriction might be $1,0.7$, and 0.2 , respectively. Zadeh's concept of linguistic variables was applied in this study to evaluate the fuzziness of students' perceptions. In order to ascertain students' perceptions of GaRA, we have decided to specify their level of satisfaction or dissatisfaction using Likert scale. Likert-type scale is a psychometric scale commonly used in researches that employ questionnaire to quantify results and perceptions. Choices usually range from strongly disagree to strongly agree for a five-point scale, and range from very strongly disagree to very strongly agree for a seven-point scale. As the category moves from one to the next, the value will increase by one unit from most negative to most positive. This allows us to measure individual attitudes, beliefs or perceptions. Likert scale is commonly used to measure different kinds of variables. A seven-point Likert provides an accurate measurement of a participant's true evaluation and more sensitive compared to a five-point Likert scale because a seven-point scale has no interpolations among respondents [28]. Scores one to three represent negative perception set, while scores five to seven represent positive perception set. Score four is considered as the midpoint which represents "neither agree nor disagree", "do not know", "no opinion", "not sure", or "undecided". Most questionnaires include midpoint on a scale. This is because, the presence or absence of a midpoint on a scale produces distortions in results obtained. Study by [29] shows that there is a tendency for respondents to choose negative ratings when a midpoint is unavailable. A scale with midpoint is appropriate as it avoids forcing respondents in choosing directions, and it does not reduce the measurement reliability and validity [30]. Nevertheless, there are limitations with the use of midpoint. Respondents have to be aware of its function so that they will not simply choose four if they are not sure about the answer. The selection of the midpoint may be reduced by increasing scale sensitivity and a number of scale options [31, 32].

$L$ is defined as a set of students' perceptions, which contain seven linguistic terms, $L_{i}=\{$ very strongly disagree, strongly disagree, disagree, neutral, agree, strongly agree, very strongly agree $\}$. Every base variable in $L_{i}$ is characterized by a membership degree, $\mu_{L i}\left(x_{j}, A\right)$, for element $x_{j}$ for item $A$ according to linguistic label $x_{j}=1,2, \cdots, 7$. The application of linguistic variable is discussed by [18] to define an evaluation of scalar measurement for seven linguistic variables. Table 2 modifies the scalar measurement for seven linguistic variables used in this study.

Table 2. Linguistic membership degree by score for each linguistic variable

\begin{tabular}{|c|c|c|c|c|c|c|c|c|}
\hline \multirow{2}{*}{ Linguistic Variable } & \multirow{2}{*}{$L_{i}$} & \multicolumn{7}{|c|}{ Score $\left(x_{j}\right)$} \\
\hline & & 1 & 2 & 3 & 4 & 5 & 6 & 7 \\
\hline Very strongly disagree & $L_{1}$ & 1.0 & 0.7 & 0.4 & 0 & 0 & 0 & 0 \\
\hline Strongly disagree & $L_{2}$ & 0.7 & 1.0 & 0.7 & 0.4 & 0 & 0 & 0 \\
\hline Disagree & $L_{3}$ & 0.4 & 0.7 & 1.0 & 0.7 & 0.4 & 0 & 0 \\
\hline Neutral & $L_{4}$ & 0 & 0.4 & 0.7 & 1.0 & 0.7 & 0.4 & 0 \\
\hline Agree & $L_{5}$ & 0 & 0 & 0.4 & 0.7 & 1.0 & 0.7 & 0.4 \\
\hline Strongly agree & $L_{6}$ & 0 & 0 & 0 & 0.4 & 0.7 & 1.0 & 0.7 \\
\hline Very strongly agree & $L_{7}$ & 0 & 0 & 0 & 0 & 0.4 & 0.7 & 1.0 \\
\hline
\end{tabular}

The linguistic membership degree represents the level of confidence of a students once they give a score for a statement. The scalar measurement shows that, for example, in linguistic variable L7, the compatibilities of scores 5,6 , and 7 with very strongly agree restriction are $0.4,0.7$, and 1.0 , respectively. Therefore, if students give the score of seven for a statement, they are $100 \%$ sure to give that score. However, as individual perceptions are fuzzy in matter, there is an ambiguity on their perceptions as there is also a probability to score five and six as well with $40 \%$ and $70 \%$, respectively. The selection of membership degree by score for each linguistic variable is arbitrarily, depends on the triangular membership function and the experienced of expert in understanding the nature of perception.

In this study, we integrated this theory with a conjoint analysis as proposed by [18] to analyze consumer preference in marketing. Fuzzy set, $R$, was used to represent the value of criteria evaluated by respondents. The membership degree of element $y_{j}, \mu_{R i}$, for the linguistic label representing attribute $A$ is defined as, 


$$
\mu_{R i}\left(y_{j}, A\right)=\sum_{i=1}^{k} W_{i} \cdot \mu_{L i}\left(x_{j}, A\right)
$$

with $\mu_{L i}\left(x_{j}, A\right)$ is a membership linguistic degree for element $x_{j}$ for item $A$ according to linguistic label $x_{j}=1,2, \cdots, n$. $A$ is an attribute or question and $n$ is the number of linguistic term. The weight vector, $W_{i}$, was calculated through the following calculation,

$$
W_{i}=\frac{w_{i}}{\sum_{i=1}^{k} w_{i}}, \quad k=1,2, \cdots, n \text {. }
$$

The membership function degree represents a fuzzy set of response of a respondent. This set was compared with the fuzzy set defined by an expert. This comparison was done using fuzzy similarity measure, Sim, as follows,

$$
\operatorname{Sim}\left(\mu_{R i}, \mu_{L j}\right)=\frac{1}{1+\sqrt{\sum_{j=1}^{n}\left(\mu_{R i}-\mu_{L j}\right)^{2}}} \quad i=1,2, \cdots, m \text { and } j=1,2, \cdots, n
$$

where $m$ is the number of attribute and $n$ is the number of linguistic term. The similarity was composed to demonstrate experts' opinions to correspond with students' opinions. The computations were executed based on the following four steps; 1) the collection and determination of the number of students answering each scale corresponding to each attribute; 2) obtaining a membership function of each element in fuzzy set $R$ by calculating a weight vector for each respondent using the $(1) ; 3$ ) obtaining similarity measures between fuzzy set $R$ and fuzzy set $L$ using (3); 4) selecting a linguistic variable $L$ which registered the highest degree of similarity. At the end of this process, we were able to determine students' preferences toward every module delivered on GaRA using maximum similarity degree.

\subsection{Membership function}

Membership function is a curve that defines how each point in the input space is mapped to a membership value between 0 and 1 . The membership function $\mu_{A}(x)$ describes a membership of the element $x$ in $X$ for fuzzy set $A$. The membership function for fuzzy set can be defined by its shape which depends on the purpose. There are many shapes of membership functions that can be considered such as triangular, trapezoidal, and Gaussian. The simplest reasonable membership functions are linear functions. Of these, the simplest is the triangular membership function. Triangular membership function has been used by [33] to solve fuzzy linear programming problem with equality constant. This type of membership function is functional due to its invariance in scale, shift, sign, and unity [34]. The function can be arbitrary, as shape can be determined from the point of view of simplicity, speed and efficiency. Therefore, triangular membership function had been used, as it has only one consensus membership degree with value 1.0 for each linguistic variable, besides it simplicity, functional and invariance for each score. The formula for triangular membership function, $f$, is,

$$
f(x: a, b, c)=\left\{\begin{array}{cc}
0 & x \leq a \\
\frac{x-a}{b-a} & a \leq x \leq b \\
\frac{c-x}{c-b} & b \leq x \leq c \\
0 & c \leq x
\end{array}\right.
$$

where $x$ is the input element in the universe of discourse, $X$, mapped to output element defined by curved values in a membership function, whereas, variables $a, b$, and $c$ represent numerical parameters for current membership function. The values for each parameter affect the stiffness of the membership shapes. Each linguistic variable has different parameter value depending on the shape of desired or considered triangle. Triangular membership shape contains three parameters which define three edges in a triangle. The membership function and its parameter are determined to each linguistic variable as in Table 3.

Table 3. Linguistic term and their membership function

\begin{tabular}{clcc}
\hline Linguistic term & & Membership function & Parameters \\
\hline Very strongly disagree & L1 & Triangular & $(-2,1,4)$ \\
Strongly disagree & L2 & Triangular & $(-1,2,5)$ \\
Disagree & L3 & Triangular & $(0,3,6)$ \\
Neutral & L4 & Triangular & $(1,4,7)$ \\
Agree & L5 & Triangular & $(2,5,8)$ \\
Strongly agree & L6 & Triangular & $(3,6,9)$ \\
Very strongly agree & L7 & Triangular & $(4,7,10)$ \\
\hline
\end{tabular}


Based on the Table 3, the second parameter represents the linguistic score for each linguistic term with $\mu_{L i}\left(x_{j}, A\right)=1$. The second parameter for each linguistic term should be at the same degree as its linguistic value. The first and third parameters show that Likert-scores have zero value of membership function for a specific linguistic term. These value represent the score that reflect the $0 \%$ of level of confidence for each linguistic term. For example, if students give the score of four (L4) for a statement, a probability to very strongly disagree (L1) and very strongly agreed (L7) to that statement at the same time is impossible. Therefore the Likert-score of four and seven have zero value of membership function for linguistic term L4. Parameter values such as $-2,-2,0,8,9$, and 10 do not exist in universal set $X$. However, these values are considered to form a symmetrical membership function. For example, the membership degree for score 1 in linguistic variable L1 is 0.7. Therefore, parameter $a$, which makes the membership degree zero in value, should be three units smaller than score 1 which is -2 . The parameters selected are arbitrarily depending on the shape of triangular membership function desired after considering the suitable membership degree for each score. We drew the triangular membership function using fuzzy logic toolbox to get better vision about a membership function as in Figure 1.

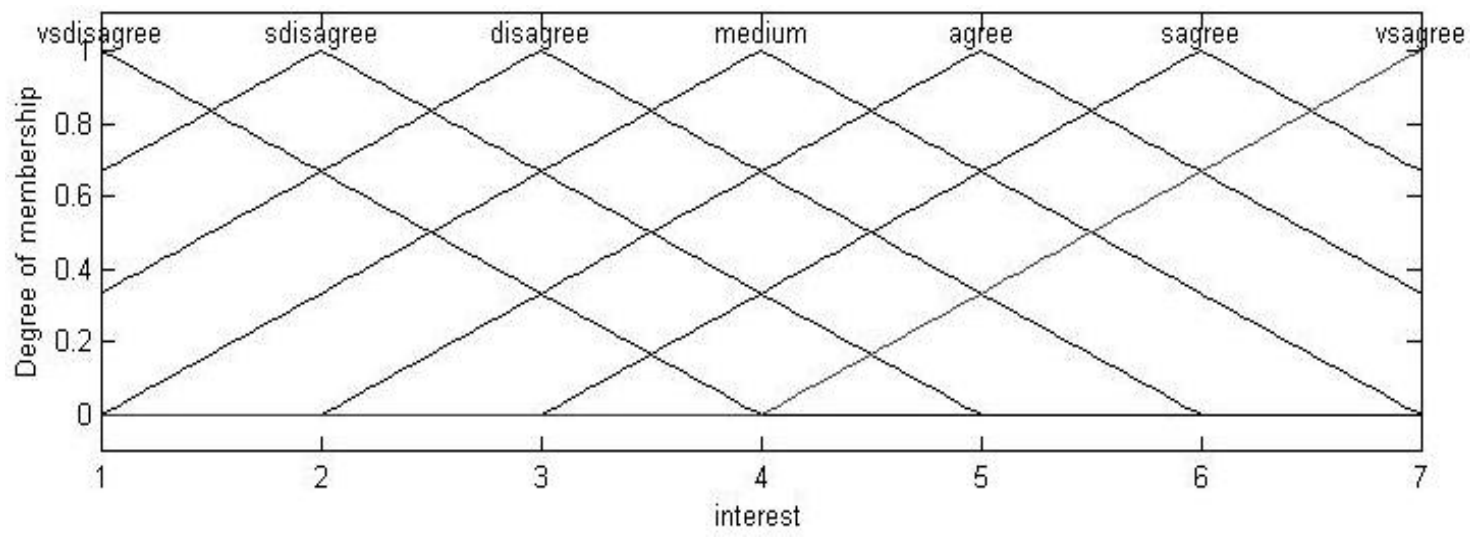

Figure 1. Triangular membership function according to parameters for interest criteria

For two different Likert scores from two different linguistic terms, there exists an intersection on the membership value. This is due to the fuzziness in perceptions, and we considered the uncertainty of every score given by students. For example, if a student scores 2 to a statement, there is a $66.7 \%$ probability that he or she very strongly diagrees and disagrees to that statement.

\section{RESULTS AND ANALYSIS}

A UKM research group called PRISMatik had organized a Mathematics Discovery Camp to increase students' affection and confidence in Mathematics using modules that implemented game and recreational approaches. The modules were delivered and developed through innovative methods in science and mathematical studies. There were 11 games conducted in this program which are KenKen \& Kakuro game, Jom Kripto, Mystery of Pascal Triangle, Paku Pakis Park \& Fractal, Balance and Stability, Sifir'a, Catapult, Mathplorace, Geometric Analysis Recreation, Ethnomathematics, and The Power of Abstraction. Each activity had its own objectives in introducing mathematical theory and knowledge. Every game had visual objects that helped to stimulate students' thinking to reach the objectives. The assignments in these games enabled students to formulate the strategies and built up their critical thinking as well as their contribution to the learning process.

Students' perceptions were collected from 1494 students of 11 secondary schools who participated in the Mathematics Discovery Camp. The statistical data was obtained from questionnaire consisting of 53\% female students and 47\% male students. Among the students, 1270 students (85\%) like Mathematics and 224 students $(15 \%)$ do not like mathematics. In their latest Mathematics examination results, 14 students $(0.9 \%)$ failed, 100 students $(6.7 \%)$ passed, 829 students $(55.5 \%)$ obtained credits and 551 students $(36.9 \%)$ received distinctions. This showed that the participants came from an average group in terms of academic achievement. The camp was held for three days and two nights with modules delivered consisting of gameplay activities in order to increase students' interest and confidence in Mathematics using GaRA 
approach. There were 11 games conducted for the participants which are KenKen \& Kakuro game (G1), Jom Kripto (G2), Mystery of Pascal Triangle (G3), Paku Pakis Park \& Fractal (G4), Balance and Stability (G5), Sifir'a (G6), Catapult (G7), Mathplorace (G8), Geometric Analysis Recreation (G9), Etnomathematics (G10), and The Power of Abstraction (G11). After completing each game, participants were asked to respond to a survey. A simple six-attribute survey was used to establish students' perceptions after completion of each module. The computation started with collecting and determining number of students answering each scale corresponding to each attribute as shown in Table 4.

Table 4. Accumulated combination value with respect to linguistic values

\begin{tabular}{cccccccc}
\hline Attribute & LI & L2 & L3 & L4 & L5 & L6 & L7 \\
\hline Interest & 29 & 17 & 29 & 77 & 98 & 245 & 930 \\
Passion & 32 & 28 & 45 & 92 & 136 & 361 & 731 \\
Solving skills & 37 & 30 & 57 & 104 & 167 & 359 & 673 \\
Effort & 16 & 14 & 22 & 73 & 105 & 301 & 889 \\
Team work & 21 & 17 & 18 & 62 & 121 & 272 & 909 \\
Extrinsic motivation & 95 & 58 & 69 & 110 & 180 & 324 & 590 \\
\hline
\end{tabular}

The data shown above is the data accumulated with respect to linguistic variable, collected for one module, which was Jom Kripto. After analysis of questionnaire, the computations of fuzzy conjoint analysis were done by calculating the weight vector, percentage of students answering each score for each attribute, followed by the membership degree, $R$, of an element $y_{j}$ for the linguistic label representing attribute $A$, using (1). Then, a similarity measurement between fuzzy set $R$ and fuzzy set $L$ was calculated using (3) before the maximum amount of similarity degree between fuzzy set $R$ and fuzzy Set $L$ for each attribute was determined as shown in Table 5.

Table 5. The value of similarity degree

\begin{tabular}{cccccccc}
\hline Attribute & LI & L2 & L3 & L4 & L5 & L6 & L7 \\
\hline Interest & 0.373 & 0.362 & 0.376 & 0.420 & 0.533 & 0.690 & 0.759 \\
Passion & 0.377 & 0.369 & 0.388 & 0.441 & 0.565 & 0.713 \\
Solving skills & 0.380 & 0.374 & 0.396 & 0.451 & 0.580 & 0.709 \\
Effort & 0.368 & 0.358 & 0.373 & 0.420 & 0.537 & 0.707 & 0.671 \\
Team work & 0.369 & 0.358 & 0.373 & 0.418 & 0.534 & 0.701 & 0.755 \\
Extrinsic motivation & 0.398 & 0.393 & 0.416 & 0.470 & 0.584 & 0.663 & 0.633 \\
\hline
\end{tabular}

Fuzzy conjoint analysis has determined the most preferred attribute that fulfilled their preference based on the similarity degree. The highest similarity degree demonstrated the maximum closeness between experts' opinions from linguistic set as in Table 2, and students' opinions as in Table 4. Based on Table 5, similarity degree of 0.362 for L2 in the first attribute was the lowest. This showed that there was a huge difference between membership degree for L2 in the first attribute (0.062) with the linguistic degree for score 2 in the first attribute $(0.7)$ as shown in Table 4 and Table 2, respectively. Therefore, the level of agreement that students have no interest in module Jom Kripto was low due to the low similarity degree.

Most attributes for Jom Kripto module achieved L6 and L7 as the maximum similarity, indicating that students stated their strong agreement that Jom Kripto module was able to increase their preference corresponding to all six attributes. Although solving skills did not achieve L7 as its maximum similarity, we still can consider that participants strongly agreed that they were able to solve the activities given. According to Table 5, the fifth attribute produced the highest rank with a value of 0.761 . This showed that this game was able to encourage students' work in group and sharing ideas to solve the problems given. The last attribute produced the lowest rank and showed that rewards did not really influence participants in solving the problems.

According to Table 2, it was believed from classical set theory that most of the students very strongly agreed (L7) that solving skills and tendency to get rewards were the significant inputs from this module with $47 \%$ and $41 \%$, respectively. However, due to the small difference in number of students with other linguistic variables and its fuzziness of students' perceptions, fuzzy conjoint analysis solved this multi-criteria problem by seeking one single consensus level with the most agreement. After the computation, it was determined that students strongly agreed (L6) that solving skills and tendency to get rewards were the significant inputs with maximum similarity of 0.709 and 0.663 , respectively. The computations were also made for the other 10 games conducted during the camp. A comparison of rank for each attribute had been done to determine the most preferred attribute that fulfilled students' preference as shown in Table 6. 


\begin{tabular}{clllllllllll}
\multicolumn{10}{c}{ Table 6. Rank of each attribute for all 11 games } \\
\hline Rank & G1 & G2 & G3 & G4 & G5 & G6 & G7 & G8 & G9 & G10 & G11 \\
\hline 1 & A4 & A5 & A1 & A1 & A5 & A1 & A1 & A1 & A1 & A3 & A1 \\
2 & A1 & A1 & A5 & A4 & A4 & A4 & A5 & A5 & A5 & A4 & A4 \\
3 & A2 & A4 & A4 & A2 & A1 & A5 & A3 & A3 & A3 & A1 & A5 \\
4 & A5 & A2 & A2 & A3 & A3 & A3 & A2 & A2 & A4 & A2 & A2 \\
5 & A3 & A3 & A3 & A5 & A2 & A2 & A4 & A4 & A2 & A3 & A3 \\
6 & A6 & A6 & A6 & A6 & A6 & A6 & A6 & A6 & A6 & A6 & A6 \\
\hline
\end{tabular}

Overall, students stated their strong agreement (L6 \& L7) that GaRA fulfilled their preference in all attributes. This level of agreement showed that GaRA was an effective program to promote affection and confidence in Mathematics. Based on Table 6, interest (A1), effort (A4) and team work (A5) were the strongest values obtained from GaRA that motivated them as these attributes fulfilled their preference almost in every module with high rank. This provided an indicator that GaRA was able to increase their level of confidence as effort and team work are the domains of confidence. Interest (A1) was the most important attribute in each game as it was ranked the best three. Lack of interest in Mathematics was one of the factors affecting difficulties in learning [2,3]. However, students put their strong agreement that interest and passion were the attributes that fulfilled their preference on Mathematics. The teaching and learning concepts from GaRA have enhanced students' interest. Positive perceptions of Mathematics and interest toward the modules were shown by the students.

Besides that, effort (F4) was the most preferred attribute in domain of confidence. Learning through game and activity was able to build students' intensity in order to identify the problem and put their effort to solve it by connecting mathematical concept and the problem statements. Interactive and relevant activities and students' persevering effort in learning process explained that GaRA fostered emotional and behavioral engagements. These engagements were important attributes towaard the effectiveness of a learning system, and hence, determined the positive results of students' performance as stated by [5].

On the other hand, team work (A5) was also one of the factors that obtained the highest rank. Students showed positive responses to peers and facilitators as they believed that team work helped them to solve the assignments in the modules. Playing provided valuable social context, communication, idea sharing and students' interpersonal skills which are the most important instruments that fostered students' engagement, and created great leaders and productive, creative and critical thinking followers as stated by [6]. These modules enabled students to share their knowledge and opinions, and hence created cooperative value which then built their confidence.

Extrinsic motivation (A6) was the most not preferred attribute as it was ranked the lowest in each module. This attribute evaluated the effect of rewards to students' intensity in solving the problems in modules. Although extrinsic motivation encouraged them to solve the problems, this attribute was in the lowest rank. Thus, this showed that rewards were not the main factor that built students' affection and confidence compared to the other attributes. Thus, the momentum showed by students throughout the programs was driven by their interest, passion, effort, and team work.

The study in preferences and conjoint formed a basis in describing students' perceptions of GaRA. The vagueness that exist in human perception was measured using FCA and the most preferred linguistic variable can be determined from the highest similarity degree. The Fuzzy conjoint analysis has shown its ability in ranking the attributes from most preferred to least preferred in the form of degree of similarity after considering the fuzziness in their perceptions in the Likert-scale regardless of the number of students involved in the study. This is an advantage in order to determine which attribute foster students' affection and confidence toward Mathematics more in compared to other attributes. Studies by [7] and [8] also showed the effectiveness of GaRA in motivating students toward Mathematics with smaller sample space. Therefore, regardless of the number of sample study, FCM is a reliable method in determining the perception and evaluating vagueness in human perceptions. Overall, it can be concluded that GaRA is effective as most students very strongly agree (L6 \& L7) that this program effectively increases their affection and confidence toward Mathematics.

\section{CONCLUSION}

Classic probability concept can be used to determine students' level of agreement for each statement from the percentage of students in answering each score.. However, this concept does not consider the fuzziness that exists in human perceptions. Therefore, FCM is the most suitable and reliable method in evaluating individual perceptions. Through this method, their level of agreement on each statement is known through the linguistic value, and hence ranks up the most preferred attribute from its maximum similarity 
degree, as FCM is able to reach each attribute from the most preferred to the least. A study on preference is important because students' feedbacks ensure the effectiveness of GaRA through effective modules. This success depends on the effectiveness of factors considered in building affection and confidence toward Mathematics as all students give the highest level of agreement (L6 \& L7) toward all factors that fulfill their preference. Thus, the method demonstrates that modules delivered in GaRA have effectively cultivated an affection and confidence in Mathematics among the participants who are average achievers in Mathematics. Apart from the degree of similarity in the selected attributes, the numerical value is able to provide extra information to educators and researchers for Mathematics education decision making.

In the future, many other factors can be considered toward students' affection and confidence. Using the same method, various multi-criteria problems can be solved, such as identifying the most essential skills in robotic system or new machinery product and, identifying the factors affecting movement of monsoon. Through this, it helps to solve the fuzziness that exists in manufacturing process and assists to develop the design, construction, and operation of engineering products.

\section{ACKNOWLEDGEMENTS}

The authors would like to acknowledge the grants received from UKM with codes ST-2017-020, GG-2017-016 and ST-2019-016.

\section{REFERENCES}

[1] A. G. Santillan, et al., "Students' Perception toward Mathematics Assessment, Temporality toward Exams, The Understanding toward Problems Associated to The Numbers and mathematical Operations in Real Life. Really are Factors Generators of Anxiety toward Mathematics?" International Journal of Developmental and Educational Psychology, vol. 1, no. 2, pp. 393-402, 2016.

[2] B. R. Acharya, "Factors Affecting Difficulties in Learning Mathematics by Mathematics Learners," International Journal of Elementary Education, vol. 6, no. 2, pp. 8-15, 2017.

[3] K. A. Gafour and A. Kurukkan, "Why High School Students Feel Mathematics Difficult? An Exploration of Affective Beliefs," Pedagogy of Teachers Education: Trends and Challenge, pp. 23-27, 2015.

[4] P. Mutodi and H. Ngirande, "The Influence of Students' Perceptions on Mathematics Performance. A Case of a Selected High School in South Africa," Mediterranean Journal of Social Science, vol. 5, no. 3, pp. 431-445, 2014.

[5] S. Gunuc, "Relationship between Students' Engagement and Their Academic Achievement," International Journal on New Trends in Education and Their Application, vol. 5, no. 4, pp. 216-231, 2014.

[6] B. Perry and S. Dockett, "Play and Mathematics," The Australian Association of Mathematics Teachers, pp. 1-4, 2007.

[7] S. S. Sofian and A. S. Rambely, "The Effectiveness of Game and Recreational Activity to Motivate High Achievers and Low Achievers: Evaluation Using Fuzzy Conjoint Analysis," AIP Conference Proceedings, vol. 1940, no. 1, p. $020128,2018$.

[8] S. S. Sofian and A. S. Rambely, "Evaluation of Students' Perceptions on Game Based Learning Program Using Fuzzy Set Conjoint Analysis," AIP Conference Proceedings, vol. 1830, no. 1, p. 050004, 2017.

[9] L. L. Thurstone, "A Method of Scaling Psychological and Educational Tests," Journal of Educational Psychology, vol. 16, pp. 433-451, 1925.

[10] L. L. Thurstone, “Attitudes Can be Measured," American Journal of Sociology, vol. 33, no. 4, pp. 529-554, 1928.

[11] R. D. Luce and J. W. Tukey, "Simultaneous Conjoint Measurement: A New Type of Foundation Measurement," Journal of Mathematical Psychology, vol. 1, no. 1, pp. 1-27, 1964.

[12] Y. X. Ho, et al., "Conjoint Measurement of Gloss and Surface Texture," Psychological Science, vol. 19, no. 2, pp. 196-204, 2008.

[13] S. A. Bodog and G. L. Florian, "Conjoint Analysis in Marketing Research," Journal of Electrical \& Electronic Engineering, vol. 5, no. 1, pp. 19-22, 2012.

[14] A. P. Singh, et al., "Analysis of Customer's Satisfaction in Public Transport Using Fuzzy Logic for Bhopal City," International Journal of Engineering and Technical Research, vol. 2, no. 12, pp. 272-275, 2014.

[15] Sushama D. and Keswani I. P., "Evaluation of Service Quality in Hospital Using Fuzzy Reasoning," International of Applied Science and Engineering Research, vol. 1, pp. 635-655, 2012.

[16] M. G. Voskoglou, “An Application of Fuzzy Logic in Measuring System Effectiveness,” International Journal of Industrial Mathematics, vol. 2, pp. 97-108, 2013.

[17] M. G. Voskoglou, "Fuzzy Logic as A Tool for Assessing Students' Knowledge and Skills," Education Science, vol. 3, pp. 208- 221, 2013.

[18] I. B. Turksen and I. A. Willson, "A Fuzzy Set of Preference Model for Consumer Choice," Fuzzy Sets and System, vol. 68, no. 3, pp. 253-266, 1994.

[19] R. H. Abiyev, et al., "Fuzzy Evaluation of Job Satisfaction of Hotel Employees," Proceedings of the $17^{\text {th }}$ International Conference of Artificial Intelligence, pp. 175-180, 2015.

[20] E. Baheri, et al., “A Fuzzy Conjoint Analysis Approach for Evaluating Credit Card Service: A case Study of Iranian Bank," African Journal of Business Management, vol. 5, no. 7, pp. 2753-2765, 2011. 
[21] L. Abdullah, et al., "Fuzzy Set Conjoint Model in Describing Students' Perceptions on Computer Algebra System Learning Environment," International Journal of Computer Science Issues, vol. 8, no. 2, pp. 92-97, 2011.

[22] L. A. Zadeh, "The Concept of a Linguistic Variable and its Application to Approximate Reasoning I," Information Sciences, vol. 8, no. 3, pp. 199-249, 1975.

[23] R. E. Bellman and L. A. Zadeh, "Decision-making in a Fuzzy Environment," Management Science, vol. 17, no. 4, pp. 141-164, 1970.

[24] P. Y. Ekel, "Fuzzy Sets and Models of Decision Making," Computers and Mathematics with Applications, vol. 44, no. 7, pp. 863-875, 2002.

[25] H. Borzecka, "Multi-criteria Decision Making Using Fuzzy Preference Relations," Operation Research and Decision, vol. 3, pp. 5-21, 2012.

[26] J. Lorkowski and V. Kreinovich, "Likert-scale Fuzzy Uncertainty from a Traditional Decision Making Viewpoint: in Incorporate Both Subjective Probability and Utility Information," Proceedings of the 2013 Joint International Fuzzy System Association World Congress and North American Fuzzy Information Proceeding Society Annual Meeting, pp. 525-530, 2013.

[27] L. A. Zadeh, "The Concept of a Linguistic Variable and its Application to Approximate Reasoning II," Information Sciences, vol. 8, no. 4, pp. 301-357, 1975.

[28] K. Finstad, "Response Interpolation and Scale Sensitivity: Evidence Against 5-point Scales," Journal of Usability Studies, vol. 5, no. 3, pp. 104-110, 2010.

[29] R. Garland, “The Mid-Point on a Rating Scale: Is It Desirable?” Marketing Bulletin, vol. 2, pp. 66-70, 1991.

[30] K. K. Tsang, "The Use of Midpoint on Likert Scale: The Implication for Educational Research," Hong Kong Teachers' Central Journal, vol. 11, pp. 121-130, 2012.

[31] M. S. Mattel and J. Jacoby, "Is There an Optimal Number of Alternatives for Likert-scale Items? Effects of Testing Time and Scale Properties," Journal of Applied Psychology, vol. 56, no. 6, pp. 506-533, 1972.

[32] R. A. Cummin and E. Gullone, "Why We Should Not Use 5-likert Scales: The Case for Subjective Quality of Life Measurement," Proceedings of Second International Conference on Quality of Life in Cities, pp. 74-93, 2000.

[33] O. Se-Ho, et al., "Solving a Fuzzy Linear Programming Problem with Triangular Membership Function," Indian Journal of Science and Technology, vol. 8, no. S9, pp. 501-505, 2015.

[34] A. Barua, et al., "Why Trapezoidal and Triangular Membership Functions Work So Well: Toward a Theoretical Explanation," Journal of Uncertain System, vol. 8, no. 3, pp. 164-168, 2014. 